\title{
Urban and rural medium voltage networks reliability assessment
}

\author{
Mohd Ikhwan Muhammad Ridzuan ${ }^{1}$ (1) - NoorFatin Farhanie Mohd Fauzi ${ }^{1}$. Nur Nabihah Rusyda Roslan ${ }^{1}$. \\ Norhafidzah Mohd Saad ${ }^{1}$
}

Received: 26 August 2019 / Accepted: 31 October 2019 / Published online: 20 January 2020

(c) Springer Nature Switzerland AG 2020

\begin{abstract}
Distribution network operators typically produce a report of network performance for all networks as a whole, by not segregating it in a different area; urban and rural networks. Although the report is sufficient, it does not represent the actual performance of each urban and rural networks. Therefore, this paper presents the configurations, parameters, and component rating for medium voltage urban and rural distribution networks. Both networks are assessed with analytical and Monte-Carlo simulation techniques. Each assessment was correlated with Energy Regulator requirements for accurate results. Urban area has better network performance due to sophisticated network automation and network configuration with $n-1$ or $n-2$ security compared to the rural area.
\end{abstract}

Keywords Urban distribution networks · Rural distribution networks · Analytical · Monte-Carlo simulation

\section{Introduction}

In each year, distribution network operators (DNOs) publish their network performance based on the number of interruption and duration of interruption. Typically most DNOs used the common reliability indices [1]; system average interruption frequency index (SAIFI), system average interruption duration index (SAIDI) and customer average interruption duration index (CAIDI). Other countries, for example, UK use different reliability indices; number of customers interrupted per 100 customers $(\mathrm{Cl})$ and number of customer minutes lost (CML). Although the terms/ names and calculation may differ, but the basis are the same; to present number of interruption (SAIFI and $\mathrm{Cl}$ ) and duration of interruption (SAIDI and CML).

Annually, DNOs have to strategieze their operation and maintenance to meet their reliability target. In the UK, DNOs annually report their actual network performance and target to Energy Regulator (OFGEM), a set of indices, namely $\mathrm{Cl}, \mathrm{CML}$ and short interruption (SI) [2]. OFGEM define a guaranteed standard of performance (GSP) to set a standard for restored supply to customers within specified period of time. Although these requirements have been set, it is not compulsory for DNOs to follow, but DNOs must be responsible to restore supply to customers within certain period of time, otherwise penalties are applied $[3,4]$. However, no rewards are given to DNOs if the requirements are satisfied. The Interruptions Incentive Scheme Performance (IIS) sets targets for $\mathrm{Cl}$ and CML. Table 1 shows the IIS for UK DNOs' and Western Power Distribution -South West (SWEST) which has missed their overall $\mathrm{Cl}$ target resulting in an overall penalty.

Before executing the strategies and setting reliability target, DNOs must simulate their network thoroughly by knowing the exact characteristics and configurations of all networks. Thus, the research/simulation should present detailed network in order to simulate network performance. Nevertheless, the medium voltage (MV) network is often represented by active and reactive power [6-8] to simplify the network due to large and complexity of upstream network. Plus, most of the customers' loads are connected within the downstream of MV network. As the

Mohd Ikhwan Muhammad Ridzuan, ikhwanr@ump.edu.my | ${ }^{1}$ Faculty of Electrical and Electronics Engineering, Universiti Malaysia Pahang, 26600 Pekan, Pahang, Malaysia.

SN Applied Sciences (2020) 2:241 | https://doi.org/10.1007/s42452-019-1612-z 
Table 1 Interruptions incentive scheme performance, 2017-2018 [5]

\begin{tabular}{lll}
\hline Distribution network operators (DNOs) & $\mathrm{Cl}$ & $\mathrm{CML}$ \\
\hline Southern electric power distribution (SSES) & 55.13 & 47.56 \\
Scottish hydro electric power distribution (SSEH) & 57.35 & 55.24 \\
SP distribution (SPD) & 41.31 & 31.19 \\
Western power distribution -south west (SWEST) & 62.04 & 42.78 \\
\hline
\end{tabular}

load aggregation is summed up from lower to higher voltage level, most of the components are being combined as one, and the characteristics of each component of aggregation are being neglected. Furthermore, the function of protection devices are ignored in the load model by the effect of aggregation. The use of protection device is crucial in network reliability assessment to segregate healthy part from faulted part of the network, significantly affecting the frequency and duration of interruption towards customers.

Figures 1 and 2 show the reliability performance of DNOs in some of European countries for years 2015 and 2016. The CEER Benchmarking Report on the Continuity of Electricity and Gas Supply 2018 [9] presented SAIFI and SAIDI indices by the segregation of voltage level; low voltage (LV), medium voltage (MV), and high voltage (HV). The HV network is a typical transmission system and the number of connected customers at point is quite small compared to LV network. Large number of customers (comprises of domestic, commercial and industrial) is connected at LV network. Eventhough the LV network is close to the customers and SAIFI/SAIDI index are related to the customers, but the fault does not occur in LV network. The MV network is basically an interconnecting path between HV and LV networks. Based on the Figs. 1 and 2, the highest portion of interruption and

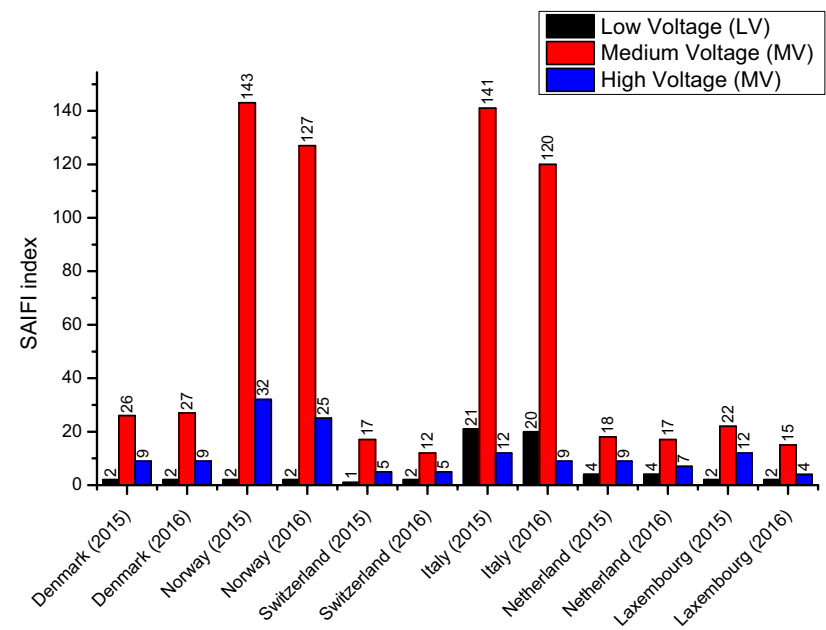

Fig. 1 SAIFI index for DNOs in European Countries [9]

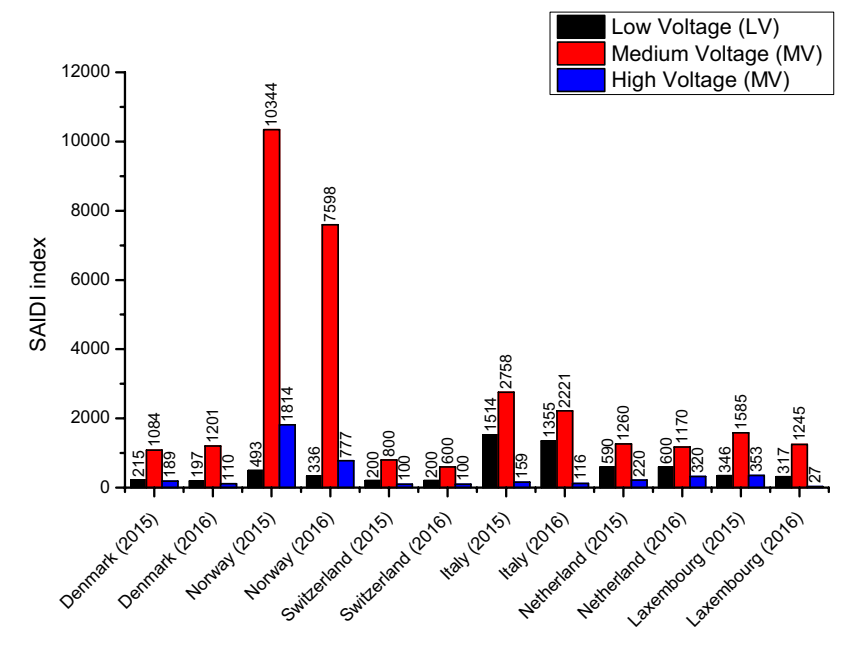

Fig. 2 SAIDI index for DNOs in European Countries [9]

duration of interruption come from MV network. The figures indicate that the focus of studies and researches should be on MV network compared to LV and HV networks.

Thus, by implementing network/load aggregation in reliability, the calculation may produce inaccurate results, as fault rates and repair times of the network components in the part of the system are incorrectly represented by the bulk load model. Therefore, presenting actual characteristics and network configuration in simulation should produce accurate results. Another concern is the separation of reliability performance of general area; urban and rural networks. Most of the reports from DNOs $[5,10,11]$ present one value of each reliability index without separating the performance of urban and rural networks. Eventhough it is normal that urban network is better than rural network, but does it always follow that pattern, and if yes, by how much? For example, in Table 1, SWEST covers number of cities like Bristol, Bath and Exeter which are close to London and Southampton, by comparing the distance from London to Dundee. The SSEH covers several sub-urban/rural areas like Dundee, Inverness, and Fort William, which are typically highland areas. Eventhough SSEH network is located in the highlands, the Cl performance of SSEH is better than SWEST. Accordingly, the paper aims to present reliability models of MV distribution networks for urban and rural areas, with its reliability data (fault rates and repair times), incorporating daily probability fault rates and load profiles, and imposed Energy Regulator requirements.

\section{Reliability assessment methodologies}

In this research, two types of approaches are used; analytical approach and Monte-Carlo Simulation (MCS) approach. The main approach is MCS, where the output is not only 
limited to mean values, but also a range of output variation. The analytical approach is used to confirm the output of MCS.

\subsection{Analytical approach}

Several assessment techniques have been developed over the past years, but the analytical calculation is prone to be used by DNOs for network planning or system security studies (e.g. n-1 or n-2 criteria), as well as for evaluating network contingencies and system capacity/reserve requirements. Even though the analytical calculation (Fig. 3 ) is a fast time approach, it cannot be directly or fully model the inherently stochastic nature of the system faults, or significant variations in fault repair time, or equally wide range of changes in system loading conditions.

The analytical calculation approach is typically related to mathematical equations, which characterize the network in terms of the specified input data, typically limiting output to one set of results, e.g. mean values of reliability indices, corresponding to specified input mean data (i.e. fault rates and mean repair time). In addition, the function of analytical approaches is limited, which cannot be integrated with load profiles, probabilitites of fault rates and network automation (e.g. reconfiguration) due to the function of analytical method; not suitable for time-sequence simulation. In other words, analytical approaches will

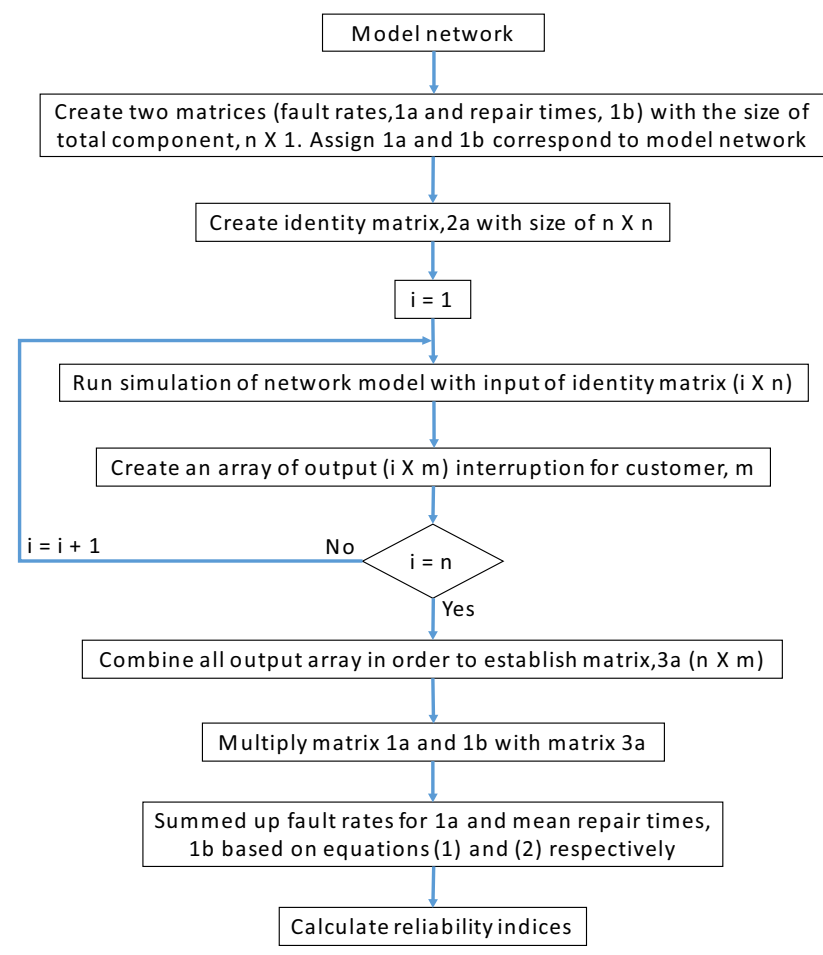

Fig. 3 Analytical approach steps always present the same set of outputs for the same set of inputs. The equation of analytical approaches which is described in [12], provides the equivalent fault rate, $\lambda_{\text {eq }}$ and mean repair time, $\mu_{\mathrm{eq}}$ for the bus where aggregate demand is connected:

$\lambda_{e q}=\sum_{i=1}^{N} \lambda_{i}$

$\mu_{e q}=\frac{1}{N} \cdot \sum_{i=1}^{N} \mu_{i}$

where $\mathrm{N}$ is a total number of power components in the equivalent part of the system, each with mean fault rate, $\lambda_{\mathrm{i}}$, and mean repair time, $\mu_{\mathrm{i}}$.

\subsection{Monte-Carlo simulation (MCS) approach}

MCS approach (Fig. 4) is able to assess network performance comprehensively with output expressed as a probability distribution (showing the range of output data). Furthermore, this approach is stochastical since it depends on random number (generated by random generator) and inherent unpredictable variation of reliability input with different possible probability distribution functions such as exponential, gamma, normal or Raleigh distribution.

Although the MCS approach is more difficult in terms of implementation (particularly in a complex, large-scale network) and a very time consuming simulation, it provides more accurate and detailed outputs than analytical approach. The type of network model and fault rates of network components are used to define which customers will be interrupted (and how frequency), whereas mean repair time of faulted components and network protection, reconfiguration and switching to alternative supply are used to estimate the duration of interruption corresponding to the supply interruption.

Based on the methods in MCS, a random variable (generated by a random generator) is assigned to an inverse cummulative distribution function to convert fault rates and mean repair time (see Table 4) into system states, time to fail (TTF), and time to repair (TTR). The system states of the network component can be modelled with a series of distribution functions; Exponential, Weibull, and Raleigh [13].

Exponential : TTF/TTR $=$ inverse $\{1-\exp (-\lambda t)\}$

Weibull : TTF/TTR $=$ inverse $\left\{1-\exp (-t / \delta)^{\beta}\right\}$

Raleigh : TTF/TTR $=$ inverse $\left\{1-\exp \left(-0.5(t / \sigma)^{2}\right)\right\}$ 


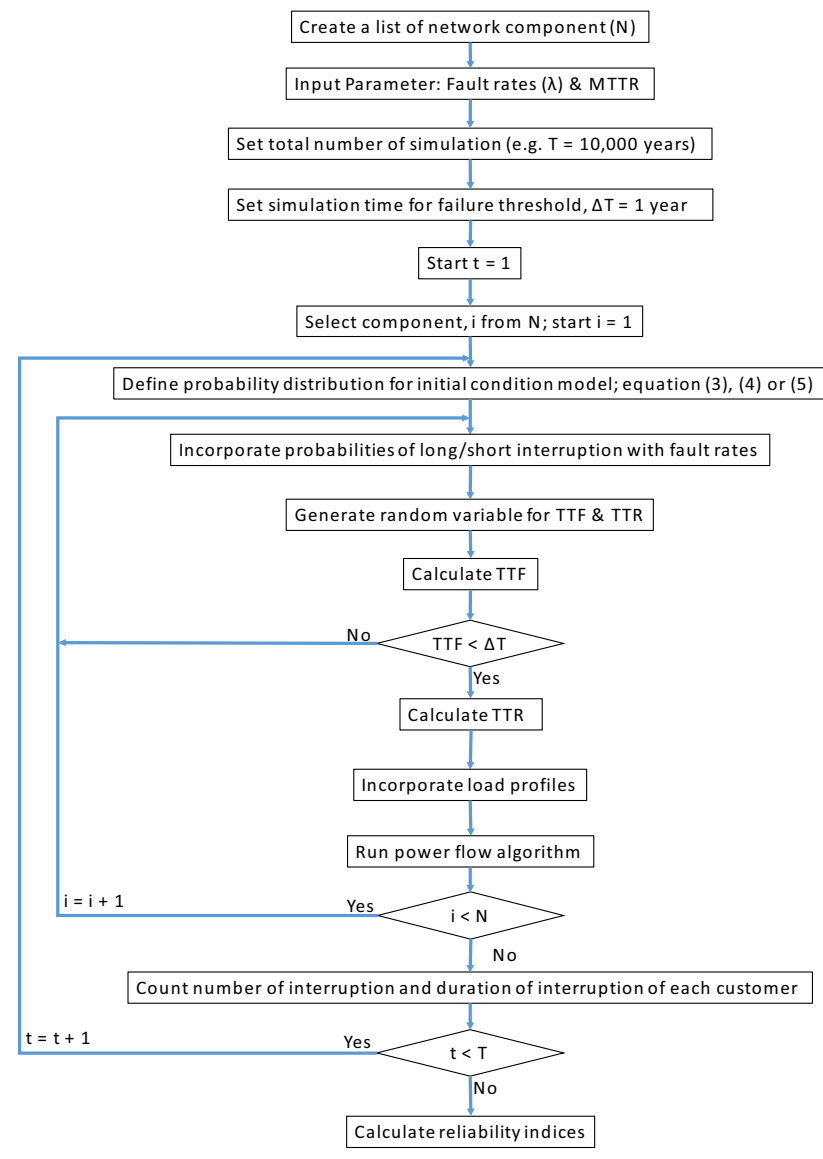

Fig. 4 MCS approach steps

To have an accurate estimation of network performance, a lot of factors need to be considered especially related to customer load and fault rates of components. In this study, the simulation correlates all network components fault rates with the probability of fault rates, while residential loads are simulated with residential load profiles, rather than maximum load. For example, if one of the $33 / 11 \mathrm{kV}$ transformers from Fig. 7 fails when load demand at the downstream of network is greater than the power rating of the other transformer, a large number of customers may be able to have continuous supply if the DNOs apply suitable corrective action. Same condition applies to component fault rates, if at that time, the probabities of fault rate is high (e.g. due to high stress/current of feeder), that component is likely to develop fault. Figures 5 and 6 show the daily probabilities of long/short interruption and typical residential load profiles, respectively.

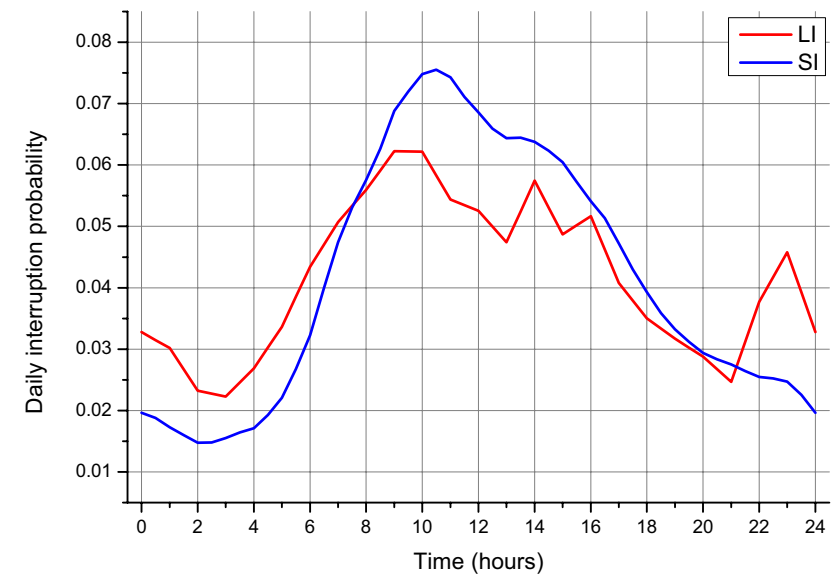

Fig. 5 Probabilities of long/short interruption (LI/SI) in 24 h [14]

\section{MV distribution network model}

The urban and rural areas are normally different in terms of geographical area, load characteristics and load density. Therefore, these considerations must be taken into account before the identification and design of MV networks. Other concerns are related economic and technical factors, such as the size of feeder, the feeder type; either underground cable or overhead lines, air- or gas-insulated switchgear and transformer ratings.

The selection of transformer depends on the load density for that area. For a load area that requires a large amount of energy and located near the path of $132 \mathrm{kV}$ transmission lines, the transformer type with transformation voltage of $132-11 \mathrm{kV}$ is favorable. In most areas of urban network, the transformer either $132 / 11$ or $33 / 11 \mathrm{kV}$ is used in substation. For this study, $33 / 11 \mathrm{kV}$ is chosen due

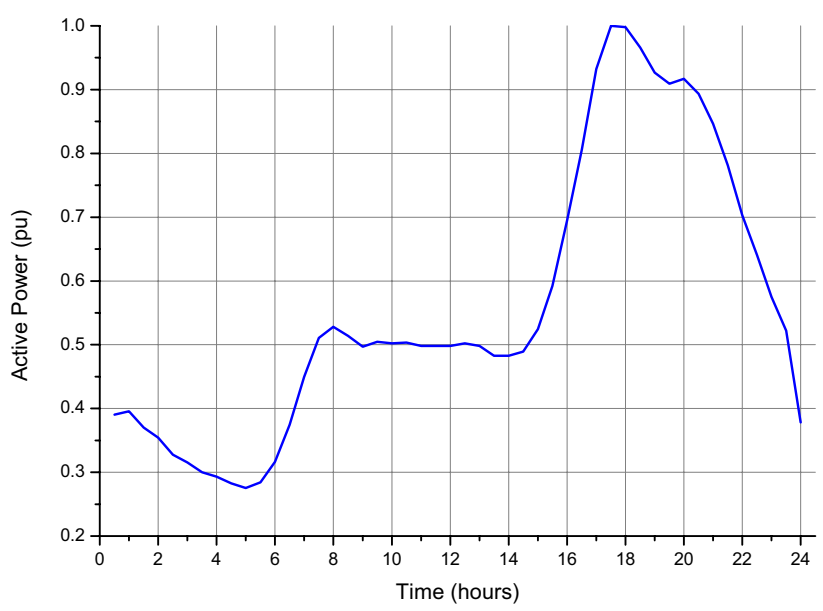

Fig. 6 Typical residential load profiles (day of maximum demand) [15] 
to the number load. The number of transformer in substation depends on the requirements of that area. Tables 2 and 3 tabulate the configurations and parameters of MV feeders, and parameters of typical 33/11 kV transformer, respectively.

\subsection{Urban (U) underground MV distribution network}

The urban area is generally populated with a densed quantity of customers, hence, space availability is limited. Therefore, network component in urban area are typically installed underground (e.g. underground cable) and most switchgears are gas-insulated types within two or three storage in tall building. The type of configuration meshes, but normally operates in radial with the support of alternative supply, either reconfiguration center or another MV primary substation ( $\mathrm{n}-1$ security), in case of fault. A reconfiguration center presents a closed-loop arrangement that connects at least two ends of feeders (closing operation of normally-open circuit breaker) during fault occurrence.
The configuration of circuit breaker from initial-end to final-end of cables are to segregate the fault part from healthy part during interruption. In Fig. 7, the lumped residential load point (dashed-circle part of $0.4 \mathrm{kV}$ ) is an illustration of detailed LV urban network [17]. The type of cables used are identified as P and Q (refer Table 2 and Fig. 7).

\subsection{Rural (R) aerial MV distribution network}

The rural network (in Fig. 8) is typically located in less-populated area and the space-availability is high. The feeders are normally overhead lines, and the switchgear insulation is air-insulated type. The network is designed in radial configuration where the power is delivered from main branch to sub-branches, then it diverges out from the sub-branches again. The MV rural network only has one $33 / 11 \mathrm{kV}$ transformer and usually do not have any back-up supplies (no $n-1$ security). Therefore, a coordinated protection arrangement between automatic recloser circuit breaker, fuse, circuit breaker, and sectionaliser are applied

Table 2 Configurations and Parameters of MV Feeders [16]

\begin{tabular}{|c|c|c|c|c|c|c|c|c|}
\hline \multicolumn{2}{|c|}{$\begin{array}{l}11 \text { kV Distribution } \\
\text { LINE TYPE }\end{array}$} & \multirow{2}{*}{$\begin{array}{l}\text { Area } \\
(\mathrm{CSA}) \\
\left(\mathrm{mm}^{2}\right)\end{array}$} & \multicolumn{2}{|l|}{$\begin{array}{l}\text { Positive sequence } \\
\mathrm{Z} / \mathrm{km}\end{array}$} & \multicolumn{2}{|c|}{$\begin{array}{l}\text { Zero-phase sequence } \\
\mathrm{Z} / \mathrm{km}\end{array}$} & \multirow[t]{2}{*}{$\begin{array}{l}\text { Susceptance } \\
\text { B/km }\end{array}$} & \multirow{2}{*}{$\begin{array}{l}\text { Max. } \\
\text { Current } \\
\mathrm{I}_{\mathrm{ph}} \\
\text { (Amps) }\end{array}$} \\
\hline Id. & Configuration & & $\begin{array}{l}\mathrm{R}_{\mathrm{ph}} / \mathrm{km} \\
\text { (p.u. on } 100 \mathrm{MVA} \text { ) }\end{array}$ & $\begin{array}{l}\mathrm{X}_{\mathrm{ph}} / \mathrm{km} \\
\text { (p.u. on } 100 \mathrm{MVA})\end{array}$ & $\begin{array}{l}\mathrm{R}_{0} / \mathrm{km} \\
\text { (p.u. on } 100 \mathrm{MVA} \text { ) }\end{array}$ & $\begin{array}{l}X_{0} / \mathrm{km} \\
\text { (p.u. on } 100 \mathrm{MVA})\end{array}$ & & \\
\hline$P$ & Underground & 185 & 0.12271 & 0.06575 & 0.85896 & 0.23011 & 0.000239536 & 415 \\
\hline Q & $\begin{array}{l}\text { Line (Cable) } \\
\text { - (3-core PICAS } \\
\text { cable (11 kV } \\
\text { screened, } \\
\text { stranded Al) } \\
\text { - (3-core XLPE } \\
\text { stranded/solid } \\
\text { Al with } 95 \text { or } \\
70 \text { mm }^{2} \\
\text { Cu wire screen) }\end{array}$ & 95 & 0.14403 & 0.06662 & 1.00824 & 0.23318 & 0.000178035 & 355 \\
\hline S & Overhead Line & 100 & 0.14658 & 0.26189 & 0.30166 & 1.31330 & 0.000012207 & 395 \\
\hline $\mathrm{T}$ & $\begin{array}{l}-\left(\text { AAAC }\left(75^{\circ} \mathrm{C}\right)\right. \\
\quad 150 \text { or } 100 \mathrm{~mm}^{2} \\
\text { Oak AL4) } \\
-\left(\text { ACSR } 54 / 9 \mathrm{~mm}^{2}\right. \\
\quad 11 \mathrm{kV})\end{array}$ & 50 & 0.21626 & 0.20694 & 0.74174 & 0.99861 & 0.000047347 & 290 \\
\hline
\end{tabular}

Table 3 Parameters of typical 33/11 kV transformer [16]

\begin{tabular}{|c|c|c|c|c|c|c|c|c|c|}
\hline \multirow[t]{2}{*}{ Sub-sector } & \multirow[t]{2}{*}{$\begin{array}{l}\text { Rating } \\
\text { (MVA) }\end{array}$} & \multirow[t]{2}{*}{$\begin{array}{l}\text { Vector } \\
\text { group }\end{array}$} & \multirow{2}{*}{$\begin{array}{l}\text { Resistance } \\
\text { R } \\
\text { (p.u. on } 100 \text { MVA) }\end{array}$} & \multirow{2}{*}{$\begin{array}{l}\text { Reactance } \\
X \\
\text { (p.u. on } 100 \mathrm{MVA} \text { ) }\end{array}$} & \multirow{2}{*}{$\begin{array}{l}\text { Zero Seq. } \\
\text { Reactance } \\
\mathrm{X}_{0} \\
\text { (p.u. on } 100 \mathrm{MVA} \text { ) }\end{array}$} & \multicolumn{2}{|c|}{$\begin{array}{l}\text { Tap Range } \\
\text { (p.u.) }\end{array}$} & \multirow{2}{*}{$\begin{array}{l}\text { Tap } \\
\text { Step } \\
\text { (p.u.) }\end{array}$} & \multirow[t]{2}{*}{$\begin{array}{l}\text { Method } \\
\text { of Earthing }\end{array}$} \\
\hline & & & & & & Min & Max & & \\
\hline Urban (U) & 15 & Dyn11 & 0.06 & 1 & 5 & 0.8 & 1.05 & 0.0143 & Resistance \\
\hline Rural (R) & 2.5 & & 0.3609 & 2.8 & 1.77 & 0.81 & 1.04 & & Solid/resistance \\
\hline
\end{tabular}




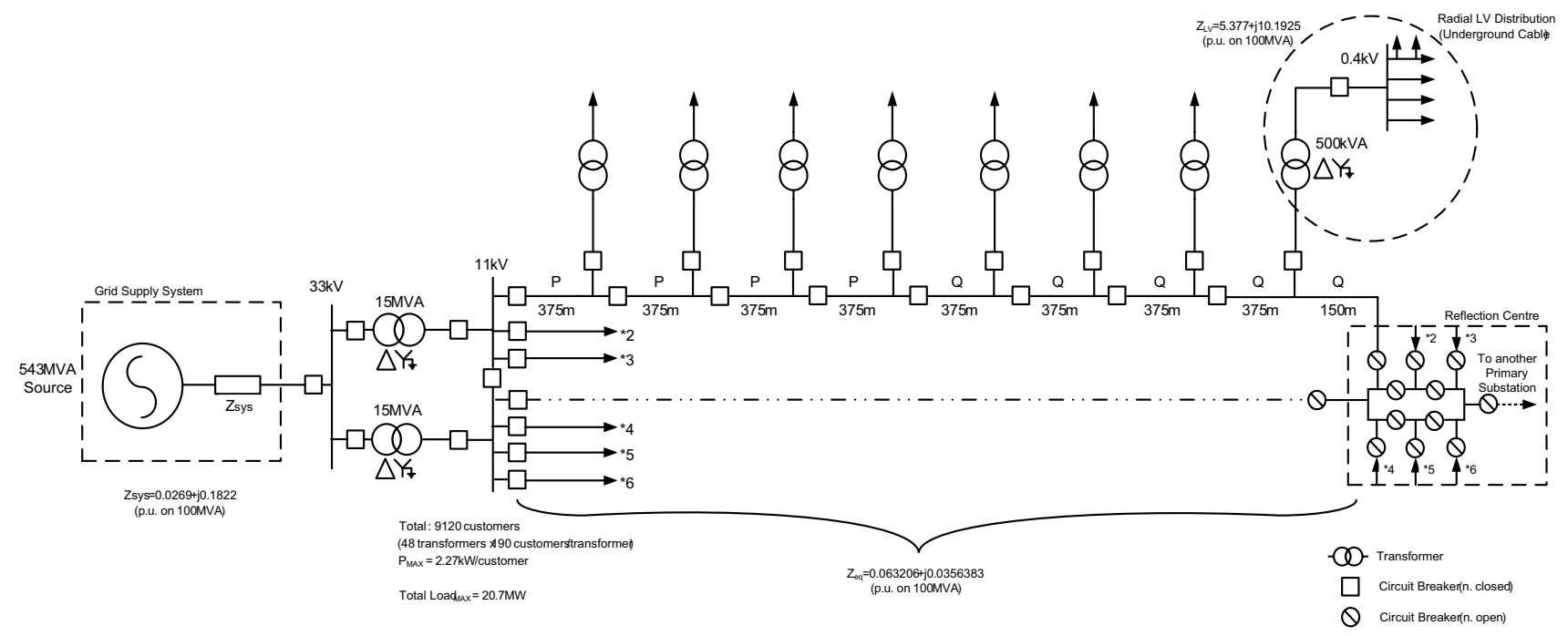

Fig. 7 33/11 kV Urban distribution network

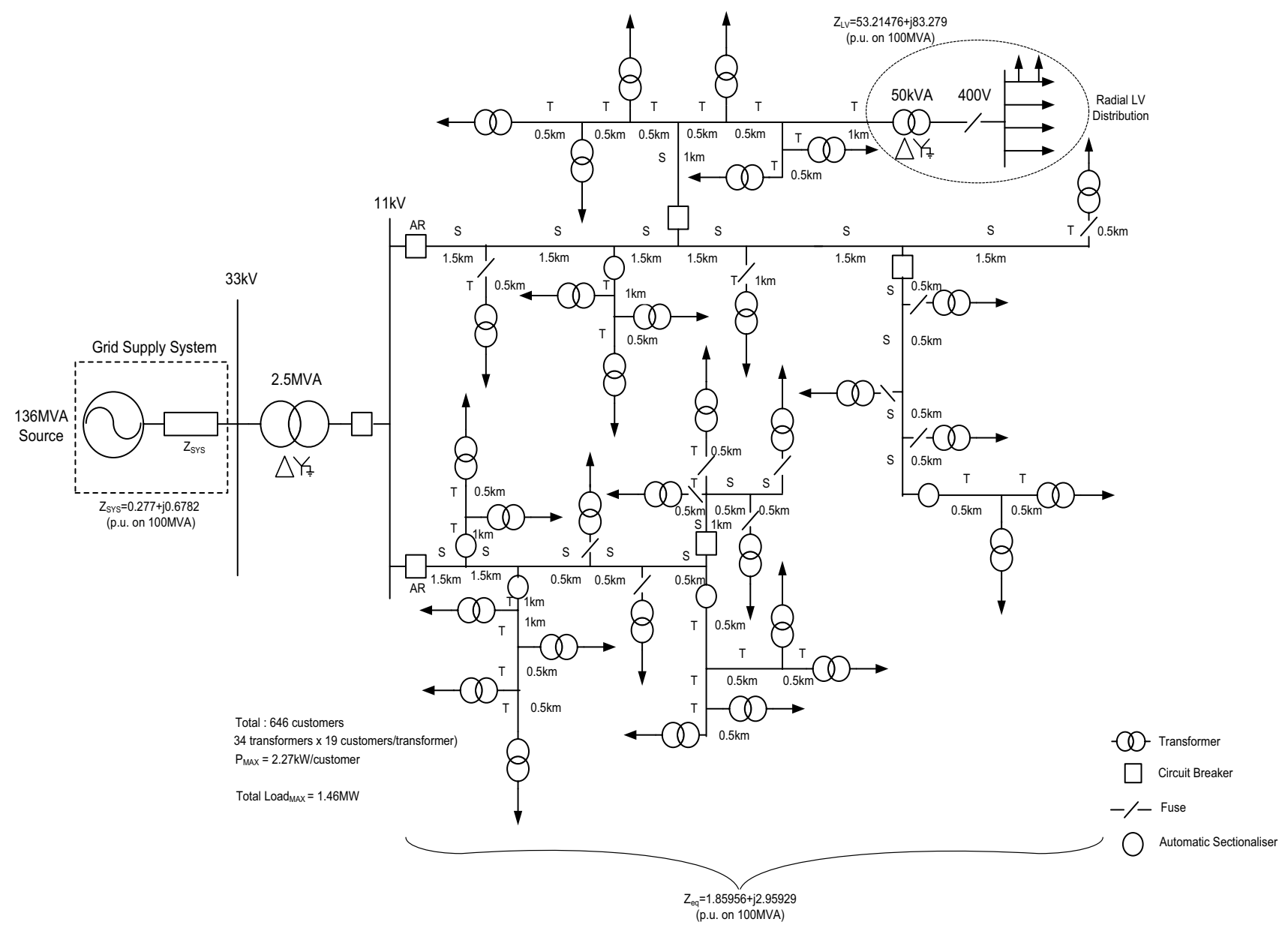

Fig. 8 33/11 kV Rural distribution network [18-20] 
to maintain continuous supply to customers. The ovaldashed part of 400 volts in Fig. 8 presents the detailed LV rural network. The type of overhead lines used are identified as $\mathrm{S}$ and $\mathrm{T}$ (refer Table 2 and Fig. 8).

\section{Simulation inputs}

\subsection{Reliability data}

Mean fault rates and mean repair time (in Table 4) are two basic inputs of network component for any reliability assessment. Mean fault rates define the probability of fault per year while mean repair times defines the time required to repair the component or replace the faulty component with a new healthy component. The networks are simulated for two reasons; result accuracy and to provide respect to the value of fault rates. First, MCS is usually a stochastic simulation, thus, by increasing the years of simulation, it will increase the accuracy of result. Second, the typical lifetime of component is 40 years. If the simulation is analysed by 40 years, it means that the fault from the fuses will not appear/arise within 40 years (40 year times with 0.0004 fault/year equal to 0.16 fault) (Fig. 9).

\section{Simulation results and discussions}

All MV distribution networks are also calculated and simulated using both approaches in Table 5. The network reconfiguration and transfer to alternative supply point ( $n-1$ security) are applied in simulation for the urban network, based on Energy Regulator's requirements. As for rural network, since the configuration of network does not have any ability to reconfigurate and transfer to alternative supply (no n-1 security), the requirements of Energy Regulator are unable to be applied. The analytical results are compared with MCS approaches, where it is simulated for a total duration of 10,000 years.

The term good reliability performance refers to having lower value reliability indices (e.g. SAIFI, SAIDI, CAIDI, etc.) as shown in Table 5; SAIFI of urban (0.1787) is better than rural (0.5083). Based on Fig. 7, urban MV network is employed with two $33 / 11 \mathrm{kV}$ transformers while in Fig. 8, rural MV network is installed with single $33 / 11 \mathrm{kV}$ transformer. By having a redundant transformer in an urban network, if one of the two 33/11 kVs experience fault, another transformer is capable to provide continuous supply to all customers depending on suitable corrective action applied and the total customer load at that moment is below average value. Another reason why urban network performance is better than rural is due to the availability of $n-1$ security for urban network.
Table 4 Mean fault rates and mean repair times of network components [21]

\begin{tabular}{|c|c|c|c|c|c|}
\hline \multirow{2}{*}{$\begin{array}{l}\text { Power component } \\
\text { Overhead Lines }\end{array}$} & \multirow{2}{*}{$\begin{array}{l}\text { Voltage level (kV) } \\
\\
<11\end{array}$} & \multicolumn{2}{|c|}{$\begin{array}{l}\text { Mean fault } \\
\text { rates } \\
\lambda_{\text {mean }} \text { (faults/ } \\
\text { year) }\end{array}$} & \multicolumn{2}{|c|}{$\begin{array}{l}\text { Mean } \\
\text { repair } \\
\text { time } \\
\mu_{\text {mean }} \\
\text { (hours/ } \\
\text { fault) }\end{array}$} \\
\hline & & 0.168 & 0.21 & 5.7 & - \\
\hline & 11 & 0.091 & 0.1 & 9.5 & - \\
\hline & 33 & 0.034 & 0.1 & 20.5 & 55 \\
\hline \multirow[t]{3}{*}{ Cables } & $<11$ & 0.159 & 0.19 & 6.9 & 85 \\
\hline & 11 & 0.051 & 0.05 & 56.2 & 48 \\
\hline & 33 & 0.034 & 0.05 & 201.6 & 128 \\
\hline \multirow[t]{3}{*}{ Trans-formers } & $11 / 0.4$ & 0.002 & 0.014 & 75 & 120 \\
\hline & $33 / 0.4$ & 0.01 & 0.014 & 205.5 & 120 \\
\hline & $33 / 11$ & 0.01 & 0.009 & 205.5 & 125 \\
\hline \multirow[t]{3}{*}{ Buses } & 0.4 & - & 0.005 & - & 24 \\
\hline & 11 & - & 0.005 & - & 120 \\
\hline & $>11$ & - & 0.08 & - & 140 \\
\hline \multirow[t]{3}{*}{ Circuit breakers } & 0.4 & - & 0.005 & - & 36 \\
\hline & 11 & 0.0033 & 0.005 & 120.9 & 48 \\
\hline & 33 & 0.0041 & - & 140 & 52 \\
\hline Fuses & $<11$ & 0.0004 & - & 35.3 & - \\
\hline
\end{tabular}

If one of the cables is faulty, the customer should receive electricity by back-up supply through network reconfiguration.

The result of Table 5 should not be compared to the exact DNOs report from the perspective of value to value basis since the test networks in this paper is only a portion of a large distribution network. The only possible factor that can be compared is the 'trending' of urban and rural network. The result in Table 5 is compared with real reliability indices (i.e. SAIFI, SAIDI, and CAIDI) to confirm the trend of load sub-sector. Table 6 presents reliability indices from Swedish benchmarking report [22], which specifies the statistics from 64 different DNOs in Sweden for 11 years (from the year 1998 to 2008). It clearly illustrates that the trend presented in Tables 5 and 6 are identical.

Another indirect comparison is between Tables 5 and 7. The connection between fault rates/MTTR and reliability indices can be found in [1]. The contribution of fault rates/MTTR should influence the reliability performance of network; higher fault rates lead to higher SAIFI. For example, in Table 7, fault rates of urban network is 0.0093 and fault rates of rural network is 0.0118 ; meaning there will be more fault originated from cables in rural area, which leads to more customers experiencing interruption of supply. The trend in Tables 5 and 7 is identical where the value of fault rates and MTTR in an urban area is smaller than rural area. The outcomes indicate that urban area has better 


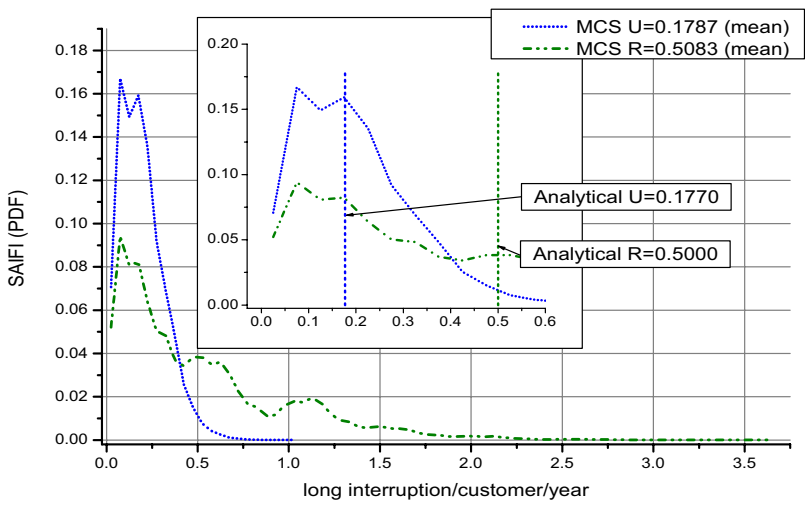

(a) System Average Interruption Frequency Index (SAIFI)

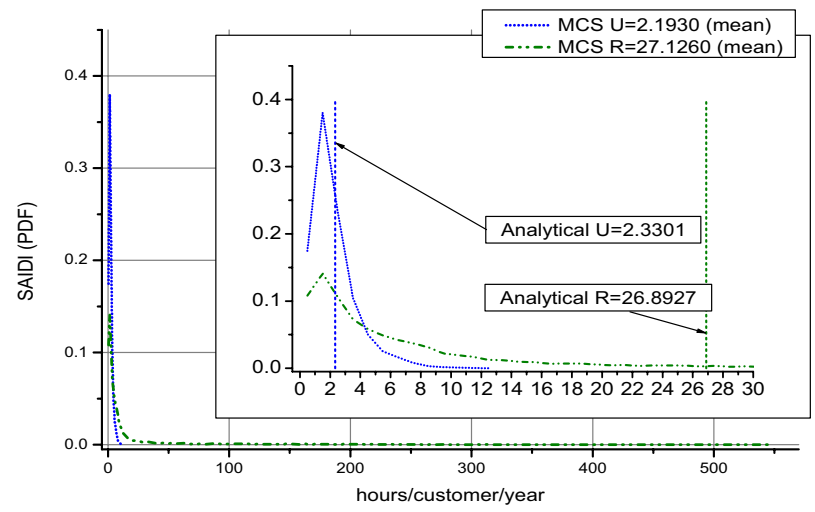

(b) System Average Interruption Duration Index (SAIDI)

Fig. 9 SAIFI, and SAIDI indices

Table 5 Analytical and MCS results

\begin{tabular}{llrr}
\hline MV networks & Indices & Analytical & \multicolumn{1}{c}{ MCS } \\
\hline Urban (U) & SAIFI & 0.1770 & 0.1787 \\
& SAIDI & 2.3301 & 2.1930 \\
& CAIDI & 13.6250 & 12.2690 \\
Rural (R) & SAIFI & 0.5000 & 0.5083 \\
& SAIDI & 26.8927 & 27.1260 \\
& CAIDI & 54.8631 & 54.8631 \\
\hline
\end{tabular}

Table 6 Swedish benchmarking report [22]

\begin{tabular}{llll}
\hline Sub-sectors & SAIFI & SAIDI & CAIDI (h) \\
\hline Urban (U) & 0.30 & 0.30 & 1.00 \\
Rural (R) & 1.65 & 6.00 & 3.64 \\
\hline
\end{tabular}

performance than rural area due to the high population of customers with various and dense load.

It can be seen in Fig. 6b, where some of the customers in rural network may experience interruption longer than $500 \mathrm{~h}$, while in urban network only less than $13 \mathrm{~h}$ of
Table 7 Reliability parameter [23]

\begin{tabular}{|c|c|c|}
\hline Components & $\begin{array}{l}\text { Mean fault rates, } \lambda \\
\text { (fault/year) }\end{array}$ & $\begin{array}{l}\text { Mean repair time, } \mu \\
\text { (h/fault) }\end{array}$ \\
\hline \multirow[t]{2}{*}{ Cables per km } & Urban-0.0093 & Urban-0.0093 \\
\hline & Rural-0.0118 & Rural-0.0118 \\
\hline \multirow[t]{2}{*}{ Transformer } & Urban-0.0005 & Urban-0.0005 \\
\hline & Rural-0.0007 & Rural-0.0007 \\
\hline \multirow[t]{2}{*}{ Circuit breaker } & Urban-0.0010 & Urban-0.0010 \\
\hline & Rural-0.0023 & Rural—0.0023 \\
\hline
\end{tabular}

interruption. In other words, urban area is equipped with sophisticated network automation and network configuration with $n-1$ or $n-2$ security for better reliability performance than rural area. Another factor that can be found in Fig. $6 \mathrm{a}$ is the probability of interruption (less than 0.5 interruption per year) for urban network is higher than rural network. This shows that urban network receives significant attention from DNOs in terms of operation and maintenance compared to rural network, due to large load density, critical load level and located nearer to maintenance team workplace.

\section{Conclusion}

The parameter of component and network configuration must be modelled and assigned correctly based on its network area; urban and rural, to assess the reliability performance accurately. The correlation of Energy Regulator requirements has set a maximum duration of interruption limit, which can be implemented in the analytical and MCS approaches for a more realistic assessment of reliability performance. These requirements are applied in network load sectors so that network reconfiguration or transfer to alternative supplies can be performed.

For each load sector, from metropolitan to remote areas, each network is modelled with detail, corresponding to the protection configurations, network configurations and parameters, and components ratings. By using the correct reliability and electrical equivalent model, large network complexity can be reduced, thus avoiding the overestimation/underestimation of reliability performance.

In conclusion, the reliability performance of urban network is better than rural network since urban network is supported by the redundancy of transformer and availability of $\mathrm{n}-1$ security (transfer of supply path through network reconfiguration). The urban network must achieve good performance due to large density of load (high number of customers) and located near to commercial and industrial loads which require reliable power supply.

\section{SN Applied Sciences}


Acknowledgements This research is supported by the Ministry of Education (FRGS/1/2018/TK04/UMP/02/16) and Universiti Malaysia Pahang under grant number RDU190186. The authors would also like to thank the Faculty of Electrical \& Electronics Engineering Universiti Malaysia Pahang for providing facilities to conduct this research and financial support throughout the process.

\section{Compliance with ethical standards}

Conflict of interest The authors declare that they have no conflict of interest.

\section{References}

1. IEEE Guide for Electric Power Distribution Reliability Indices (2012) IEEE Std 1366, p 43

2. Electricity Distribution Quality of Service Report 2008/09 (2009) Office of Gas and Electricity Markets (OFGEM) Annual Report

3. The Electricity Standard of Performance Regulations Statutory Instruments (2010) In Standard No. 698, Office of Gas and Electricity Markets (OFGEM)

4. The Electricity (Standards of Performance) Regulations (2015) Standard No. 699, Office of Gas and Electricity Markets (OFGEM)

5. RIIO-ED1 Annual Report for the Distribution Network Operators Performances in 2017-18 (2018) Office of Gas and Electricity Markets (OFGEM), London, pp 1-52

6. Jeong $M-G$ et al (2017) Optimal voltage control using an equivalent model of a low-voltage network accommodating inverterinterfaced distributed generators. Energies 10(8):1180

7. Afandi I, Ciufo P, Agalgaonkar A, Perera S (2018) A holistic approach for integrated volt/var control in MV and LV networks. Electr Power Syst Res 165:9-17

8. Di Fazio A, Russo M, De Santis M, Di Fazio AR, Russo M, De Santis $M$ (2019) Zoning evaluation for voltage optimization in distribution networks with distributed energy resources. Energies 12(3):390

9. CEER (2018) CEER Benchmarking Report 6.1 on the Continuity of Electricity and Gas Supply, p 84

10. Energy Commission (2016) Performance Standard of Electricity Supply Services of TNB, pp 1-8

11. SP Energy Networks Distribution Annual Report 2017/18 (2018)
12. Billinton R, Wang $P$ (1998) Reliability-network-equivalent approach to distribution-system-reliability evaluation. IEE Proc Gener Transm Distrib 145(2):149-153

13. Muhammad Ridzuan M, Djokic S, Muhammad Ridzuan MI, Djokic SZ (2019) Energy regulator supply restoration time. Energies 12(6):1051

14. Djokic SZ, Ilie I-S, Hernando-Gil I (2014) Theoretical interruption model for reliability assessment of power supply systems. IET Gener Transm Distrib 8(4):670-681

15. Ridzuan MIM, Gil IH, Djokic SZ, Langella R, TestaA (2014) Incorporating regulator requirements in reliability analysis of smart grid. Part 1: Input data and models. In: Innovative smart grid technologies (ISGT) European 2014 conference

16. Hernando-Gil I, Hayes B, Collin A, Djokic S (2013) Distribution network equivalents for reliability analysis. Part 1: aggregation methodology. IEEE PES ISGT Eur 2013:1-5

17. Ridzuan MIM, Hernando-Gil I, Djokic S, Langella R, Testa A (2014) Incorporating regulator requirements in reliability analysis of smart grids. Part 1: Input data and models. In: Innovative smart grid technologies conference Europe (ISGT-Europe), 2014, pp $1-5$

18. Lakervi E, Holmes EJ (2003) Electricity distribution network design, 2nd edn. Institution of Engineering and Technology (IET), London

19. Northen Powergrid. Code of practice for the protection and control of HV circuits (DSS/007/010)

20. Charlesworth D (2007) Primary network design manual. In: EON central network, pp 1-118

21. Muhammad Ridzuan MI, Hernando-Gil I, Djokic S (2018) Reliability analysis on protection devices inclusion in LV residential distribution network. J Telecommun Electron Comput Eng 10(1-2)

22. Torstensson D, Bollen M, Kolessar R (2011) The Swedish benchmarking report on continuity of supply. In: 21 st International conference on electricity distribution (CIRED), no. 233, pp 1-4

23. Meeuwsen J (1998) Reliability evaluation of electric transmission and distribution systems

Publisher's Note Springer Nature remains neutral with regard to jurisdictional claims in published maps and institutional affiliations. 